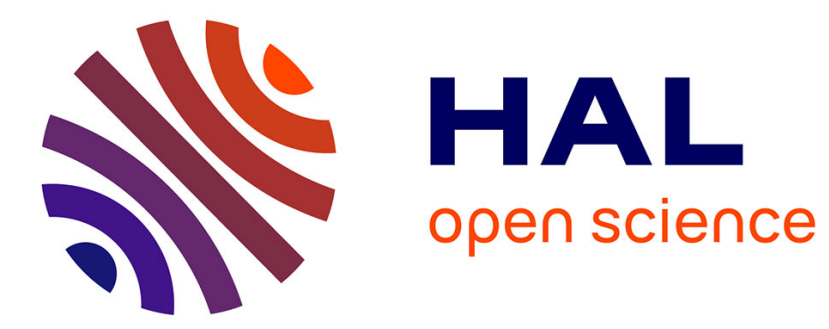

\title{
Optic flow-based robotics
}

Julien Serres, Franck Ruffier

\section{To cite this version:}

Julien Serres, Franck Ruffier. Optic flow-based robotics. John Wiley \& Sons, Inc., pp.1-14, 2016, 10.1002/047134608X.W8321 . hal-02294347

\section{HAL Id: hal-02294347 https://hal-amu.archives-ouvertes.fr/hal-02294347}

Submitted on 23 Sep 2019

HAL is a multi-disciplinary open access archive for the deposit and dissemination of scientific research documents, whether they are published or not. The documents may come from teaching and research institutions in France or abroad, or from public or private research centers.
L'archive ouverte pluridisciplinaire HAL, est destinée au dépôt et à la diffusion de documents scientifiques de niveau recherche, publiés ou non, émanant des établissements d'enseignement et de recherche français ou étrangers, des laboratoires publics ou privés. 


\title{
Optic flow-based robotics
}

\author{
Julien SERRES and Franck RUFFIER \\ \{julien.serres, franck.ruffier\}@univ-amu.fr \\ Aix-Marseille University, CNRS, ISM UMR 7287, 13288, \\ Marseille, France
}

April 26, 2016

\begin{abstract}
Flying insects and birds are able to fly smartly in an unpredictable environment. Many animals have been found to rely mainly on optic flow. Optic flow can be defined as the vector field of the apparent motion of objects, surfaces, and edges in a visual scene generated by the relative motion between an observer (an eye or a camera) and the scene. Optic flow is particularly interesting for short-range navigation because it depends on the ratio between (i) the relative linear speed of the visual scene with respect to the observer and (ii) the distance of the observer from obstacles in the surrounding environment, this does not require any measurement of either speed or distance. Optic flow is therefore suitable for various navigational tasks such as: takeoff or landing along vertical or longitudinal axes, terrain following, speed control in a cluttered environment, lateral and frontal obstacle avoidance and visual odometry. Our survey focuses on feedback-loops which use optic flow to control robots in the same way as the Gibsonian approach which sometimes enhances robot perception, by a distance or speed measurement, even though the direct measurement of distance or linear speed does not exist in flying insects and birds. Optic flow is likely to be one of the most important visual cues that could be used during the next decade to enhance robot reactivity in unpredictable environments. Conversely, the biorobotic approach can therefore help to better understand how flying animals can move smartly in such an environment.
\end{abstract}

Keywords. Short-range navigation, mapless navigation, GPS-denied environment, biorobotics, bionics. 


\section{INTRODUCTION}

\section{Why use the optic flow in Robotics?}

Robots are today capable of accurately evaluating their position and orientation in the three dimensions using sensory systems such as the Global Positioning System (GPS) and inertial navigation systems. This is very efficient for longrange navigation at high altitude far above the ground (several $\mathrm{km}$ ), without obstacles around them, such as an airplane in cruising flight. Nevertheless, the expanding set of roles for robots increasingly calls for them to operate close to obstacles $(<1 \mathrm{~m})$, and in both indoor and outdoor environments containing obstacles in all directions. Close to the ground, it is more difficult for a robot to get its accurate position with respect to obstacles when it is continually on the move. The robot can have difficulties receiving the GPS signal in GPSdenied or cluttered environments but it still has to pursue its mission. Despite such difficulties, the robot has to pick up the 3D structure of the surrounding environment to avoid obstacles and accomplish its mission. At such a short distance from obstacles $(<1 \mathrm{~m})$, the environment is unpredictable: it is obviously very difficult to map the entire environment in $3 \mathrm{D}$ at such a scale. A more efficient approach would consist of the robot continuously using local information to avoid obstacles whilst waiting for global information to pursue its mission. Most of the time the use of emissive proximity sensors such as ultrasonic or laser range finders, radar, or scanning LIght Detecting And Ranging (LIDAR) has been considered for this purpose. Such emissive sensors can be bulky, stealth-compromising, high energy, and low-bandwidth - compromising their utility for tiny and insect-like robots. It is well known that flying insects such as flies and bees are sensitive to optic flow ([1]; 2]; [3]; 4]; [5]) and in particular, they are able to measure optic flow of the surroundings irrespective of the spatial texture and contrast $([6] ; 7] ; 8] ; 9])$, and some of their neurones respond monotonically to optic flow ([2]; [10]). Consequently, there are considerable benefits to be gained by designing guidance systems for robots that utilize passive sensing, such as vision, and more precisely motion vision, because a visual system with poor acuity using low computational resources can be implemented onboard robots.

\section{What is the optic flow?}

The optic flow vector field perceived by a robot depends in particular on the structure of the environment ([11]; 12]; [13]; 14]; 15]; 2]). The optic flow can be defined by a vector field of the apparent motion of objects, surfaces, and edges in a visual scene caused by the relative motion between an agent and the 
scene (Fig 1 ). The optic flow field $\vec{\omega}(1)$ is the combination of two optic flow components: a translational optic flow $\overrightarrow{\omega_{T}}(\mathrm{Fig}, 1 \mathrm{a})$ and a rotational optic flow $\overrightarrow{\omega_{R}}($ Fig, $1 \mathrm{~b})([15])$.
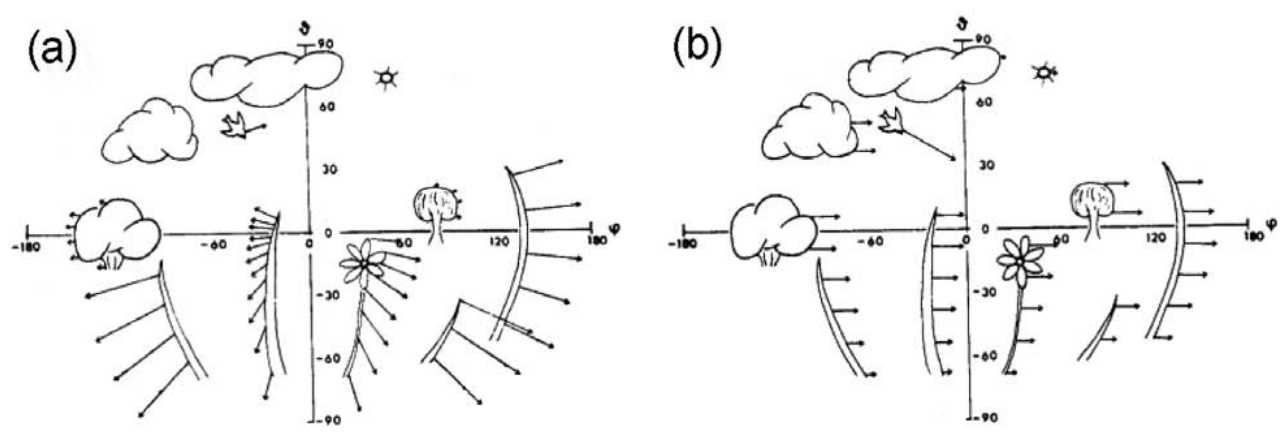

Figure 1: Optic flow field for an agent moving straight ahead in (a), or an agent rotating around its vertical axis in (b). Adapted from [16] (C)Springer.

$$
\vec{\omega}=\overrightarrow{\omega_{T}}+\overrightarrow{\omega_{R}}
$$

Rotational optic flow depends only on agent's own rotations. However in the horizontal plane, translational optic flow, which describes the front-to-back motion occurring when the robot moves forward, depends on the ratio between the relative linear speed $V$ and the distance $D_{\varphi}$ from the contrasting objects of the environment, and the angle $\varphi$ between the gaze direction and the speed vector $(2)$.

$$
\omega_{T}=\frac{V}{D_{\varphi}} \cdot \sin (\varphi)
$$

Translational optic flow ((2) ) is particularly interesting for short-range navigation because it depends on the ratio between (i) the relative linear speed of an object in the scene with respect to the agent and (ii) the distance from obstacles in the surrounding environment, this does not require either speed or distance measurement ((2)). Different optic flow sensors have been developed for short-range navigation purposes including hardware ([17]; 18]; 19]) and software implementation [19].

\section{Robot-Fly and Bee-Bot: the pioneers of the optic flow-based robots}

In the 80s, robust optic flow computation methods were developed (e.g. Lucas \& Kanade's method (1981) [20] and Horn \& Schunck's method (1981) [21]), as 
were custom-made optic flow sensors [22] allowing robots to be endowed with motion vision. The first robot built using optic flow for short-range navigation was the Robot-Fly (in French "robot-mouche", Fig.2p); 23]; 24]). The RobotFly always moved at the same constant speed across an obstacle forest, sensing the world during an elementary translation step (Fig $\sqrt[3]{3}$. This step-by-step locomotion was inspired by the freely flying insects' locomotion mode, which has been found to usually consist of straight flight sequences (lasting $50-200 \mathrm{~ms}$ ) interspersed with rapid turns termed saccades ([25]; 26]; 27]; 28]). Intersaccadic sequences, in which the Robot-Fly moved in a purely translation mode (length 10cm; duration 200ms), enable its 116-pixel artificial panoramic eye to assess the purely translational optic flow, which depends on parameters including the robot's speed $(50 \mathrm{~cm} / \mathrm{s})$ and its distance from obstacles. At the end of each step, the Robot-Fly panoramic eye had therefore detected the proximity of the local obstacles. An analog-electronics based fusion occurs between the spatial information concerning the angular bearing of the goal and the angular bearings of the obstacles, and ensures that the robot's next heading will be the as close as possible to the goal while avoiding obstacles. These short-lived proximity cues were updated after each step, the Robot-Fly did not rely on nor build any metric representation of its environment. The Robot-Fly was particularly reactive to local obstacles because its panoramic eye was able to measure a frontal optic flow in a range of $\left[6^{\circ} / s ; 135^{\circ} / s\right]$ and a lateral optic flow in a range of $\left[25^{\circ} / s ; 574^{\circ} / \mathrm{s}\right]$, corresponding to 1.4 -decade of optic flow range. The second one was called the Bee-Bot and built by Coombs and Roberts in 1992 ([29]; 30]). The Bee-Bot took inspiration from the bee's centering behavior $([7 ;$; 8 ) in its visual control system. Bee-Bot was fitted with a pair of wide-angle cameras (with a $256 \times 256$-pixel image, frame rate $10 \mathrm{~Hz}$ ) with a $115^{\circ}$ field of view. The pair of cameras were both facing obliquely to left and right at about $30^{\circ}$ forward of side-looking. The optic flow was basically computed using a gradient method, and could be measured in the range of $\left[4^{\circ} / s ; 20^{\circ} / \mathrm{s}\right]$ therefore limiting the Bee-Bot's speed to about $10 \mathrm{~cm} / \mathrm{s}$ during experiments. The maximal optic flow value taken from each peripheral field indicated the nearest obstacles to right and left, consequently by balancing the optic flow on both sides, the Bee-Bot was able to center its trajectory between obstacles. 25 years ago, both Robot-Fly and Bee-Bot demonstrated their ability to use visual information to avoid local obstacles without any global representation of the environment. Both robots are fitted with very low resolution visual systems, but use distinct optic flow-based strategies to assess proximity information from their environment. 


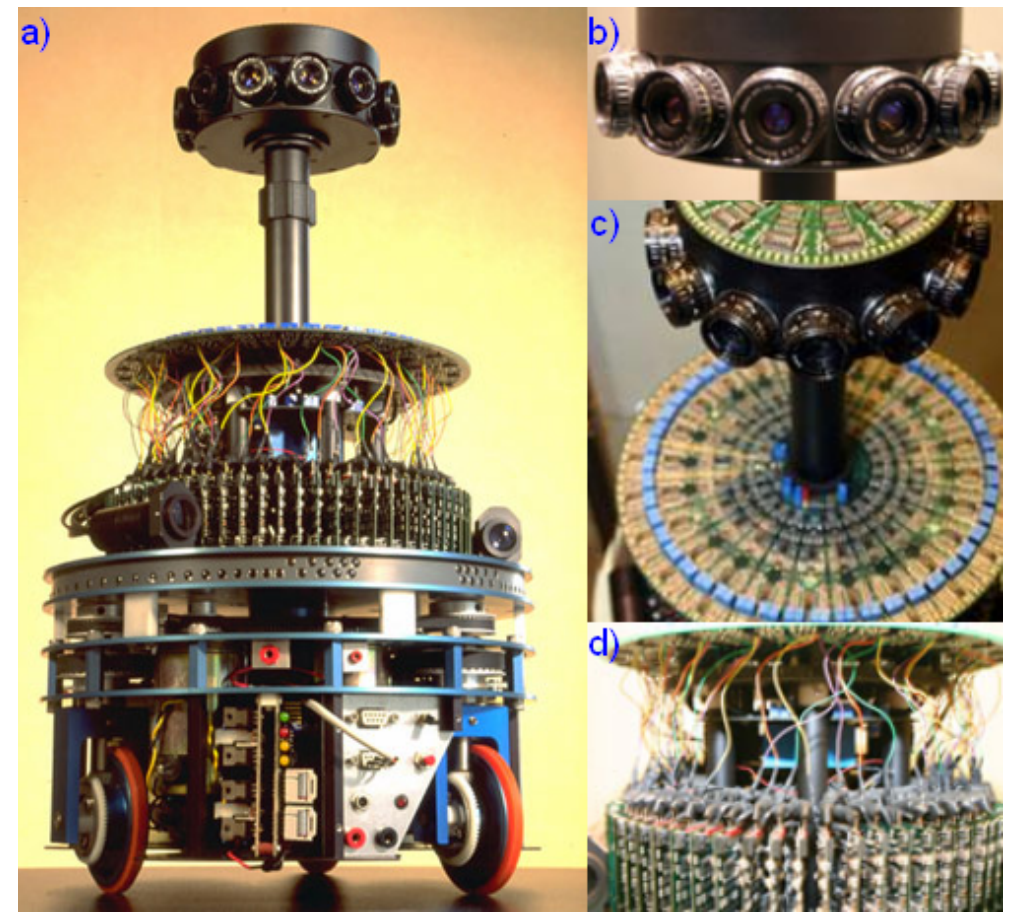

Figure 2: (a) Robot-Fly (in French: "Robot-mouche") with a visual system composed of a compound eye (visible at half-height) for obstacle avoidance. This $12 \mathrm{~kg}$ three-wheeled robot, which was completed in 1991 (23]; 24]) is fully autonomous as regards its processing and power resources. (b) and (c) A target seeker for detecting the light source serving as a goal. (d) Despite its small number (116) of pixels, this robot can avoid obstacles at a relatively high speed $(50 \mathrm{~cm} / \mathrm{s})$ by reacting to the optic flow generated by its own locomotion. It carries a set (114) of local motion sensors 22], each of which is inspired by the fly's Elementary Motion Detector (EMD). (C)CNRS Photolibrary / Nicolas Franceschini.

\section{The chicken-and-egg problem of the translational optic flow}

A given value of translational optic flow is a kind of chicken-and-egg problem (2), because an infinite number of couples (speed; distance) lead to the same speed/distance ratio, i.e. the same optic flow value. For instance, an optic flow value of $1 \mathrm{rad} / \mathrm{s}$ (i.e., $57^{\circ} / \mathrm{s}$ ) can be generated by a robot moving at $1 \mathrm{~m} / \mathrm{s}$ at $1 \mathrm{~m}$ from an obstacle, or moving at $2 \mathrm{~m} / \mathrm{s}$ at $2 \mathrm{~m}$ from an obstacle, and so on. 


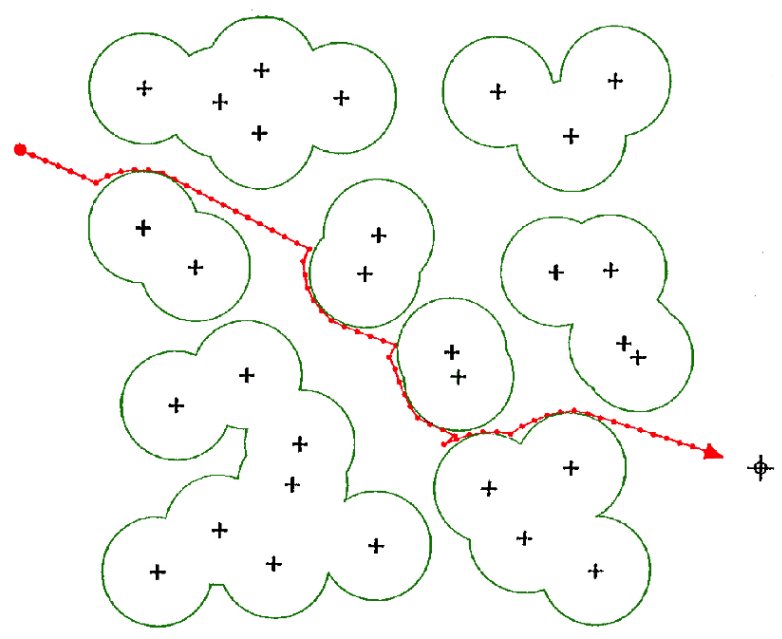

Figure 3: Simulation of the Robot-Fly fitted with a bio-inspired panoramic eye for obstacle avoidance and a light target detector to reach the goal by positive phototaxis behavior. Robot-Fly is able to navigate across an obstacle forest [24]. Courtesy from Nicolas Franceschini / Jean-Marc Pichon / Christian Blanes.

To get around the optic flow chicken-and-egg problem, roboticists introduced the assumption prevailing in those days that robots have to measure their own speed (by using a tachymeter on wheels: 223] [24], a GPS unit: [31]; [32], a custom-built pitot tube [33]), or by whatever means in simulation [34] in order to assess the obstacles' distances, then to avoid them. However, flying animals are not able to directly measure their true ground speed or their distance from an obstacle. As far as we know, insects do not solve the optic flow chicken-andegg problem but instead use strategies directly based on optic flow criterions that we have called optic flow regulators ([35]; 36]; [37]).

\section{OPTIC FLOW-BASED STRATEGY IN THE HORIZONTAL PLANE}

\section{Optic flow balance strategy}

25 years ago, the optic flow balance strategy was put forward to explain the bee's centering behavior ([7]; 8$]$ ). This quite simple strategy was quickly applied in the field of Robotics [29]. The idea is basically to control the heading by 
means of a heading error $\Delta \Psi(3)$ as a function of an optic flow error $\left|\omega_{L}\right|-\left|\omega_{R}\right|$, where $\omega_{L}$ and $\omega_{R}$ are respectively the maximum optic flow amplitude coming from the left and the right part of the field of view, and $K_{\Psi}$ is the gain of the proportional controller (Fig,4).

$$
\Delta \Psi=K_{\Psi} \cdot\left(\left|\omega_{L}\right|-\left|\omega_{R}\right|\right)
$$

The heading error $\Delta \Psi$ will therefore become, by linearizing (3), proportional to the distance error $\Delta D$ with respect to a corridor midline (4).

$$
\Delta \Psi=K_{\Psi} \cdot\left(\left|\frac{V_{f}}{D_{L}}\right|-\left|\frac{V_{f}}{D_{R}}\right|\right)=4 \cdot K_{\Psi} \cdot V_{f} \cdot \frac{\Delta D}{D^{2}}
$$

Where $V_{f}$ represents the robot's forward speed, $D$ represents the local corridor width, and $D_{L}$ and $D_{R}$ are respectively the distance from the left and right walls. Consequently, equalizing the two lateral optic flows $\left(\omega_{L} \approx \omega_{R}\right)$ with (3) may lead the robot to follow the corridor midline by canceling the distance error $\Delta D$ with respect to the corridor midline (4). Interestingly, in the particular case of a straight corridor, the robot will reach the corridor midline while its heading is also aligned with the corridor axis (Fig 4 4 ). The reality of experimental robotic trajectories shows that an optic flow balance trajectory always leads to an oscillatory trajectory around the corridor midline, because the robot is constantly avoiding two walls (the right one, or the left one). The optic flow balance strategy has been implemented on-board many terrestrial wheeled robots ([29]; [38]; [39]; [40]; 44]; 42]; 43] ; [44]; 45]; 46]; [47]; [48] ; 49]), and many aerial vehicles (blimp: [50]; simulated hovercraft: [51] ; simulated helicopter: [52] ; helicopter: [47]; unmanned air vehicle: [53]). In each experiment the robot was able to follow the corridor or canyon midline with more or less oscillations either in indoor or in outdoor conditions, because actually the equilibrium position along the corridor midline is obtained by jointly avoiding the two lateral walls. Simple proportional controllers (gain in (4)) were used to control the robot's heading ([29]; [40]; 41]; [52]), or even lead controllers to stabilize the robot in case of lateral obstacles ([39]). However, using an integral action in the controller would result in destabilizing the robot's behavior ([39]). Works on optimizing the optic flow balance strategy demonstrated that the robot's centering behavior was optimum with respect to the maximum force restoring the robot toward the corridor axis for a pair of cameras faced obliquely at $45^{\circ}$ forward of side-looking ([54]; [33]). However, if the optic flow balance strategy alone can keep a robot near the midline of a straight corridor or an urban straight canyon, it cannot be used to negotiate T-junctions or L-junctions without including another visual modality to detect 
frontal obstacles ([40; 477]). To get around this difficulty and to allow the robot to detect imminent collision and to trigger an avoidance maneuver with a higher level of priority than the optic flow balance strategy, a frontal obstacle system must be implemented on the basis of the average time-to-contact [55] in the central part of the field of view ([40]; [41]), or on the basis of stereo vision [47. In both cases, if the time-to-contact (or the frontal distance) is below a critical value, the robot will stop and turn away to avoid the obstacle. If the robot moves at a constant forward speed inside a corridor, the visual motion requires measuring a wide range of optic flow to equalize the two lateral optic flows because the corridor could be narrow, thus generating a high optic flow value, or wide, generating a low optic flow value. That's why controlling the speed of the robot according to the width of the corridor, allows the roboticist to limit the range of the optic flow measurement around the best sensitivity of the visual motion sensors. However, attempting to balance the two lateral optic flows would make a robot rush into any openings in a wall, since an opening offers zero optic flow (if $\Delta D \rightarrow+\infty$ in (4), then $\Delta \Psi \rightarrow+\infty$ ). To solve this problem, some authors proposed switching to a "wall-following behavior" based on keeping the unilateral optic flow constant, coupled with a constant speed when the average of the two lateral optic flows becomes larger than a given threshold [41] (Fig,4 ) or when one of the lateral optic flows is at a zero value [39].

\section{Keeping the unilateral optic flow constant coupled with a constant speed}

Keeping the unilateral optic flow constant and coupling this with a constant forward speed was tested on-board many robots encountering lateral openings along a corridor ([39] and Fig.5p; 41] and Fig.5b; [56]; [57]) or moving inside a square arena ([58] and Fig [5c). Keeping the unilateral optic flow constant consists of using one of the two lateral optic flows to follow the nearest wall to the robot by maintaining a unilateral optic flow constant. The desired optic flow can therefore be considered as a pre-specified distance set-point. This strategy was applied on wheeled robots whose speed was adjusted to a relatively low velocity $(8 \mathrm{~cm} / \mathrm{s}$ in $[39] ; 12 \mathrm{~cm} / \mathrm{s}$ in [41]; $10 \mathrm{~cm} / \mathrm{s}$ in [58]), or on a simulated hovercraft moving at $1 \mathrm{~m} / \mathrm{s}$ [56]; 57]). To stabilize the unilateral optic flow regulation, lead controllers were mainly employed to limit the robot's oscillations ([39]; 41]; [58]; [56]; [57]) because lateral dynamics piloted by the robot's heading introduced two successive integrations significantly reducing the phase margin of the regulation loop. However, controlling the robot's lateral translations by yawing introduced a rotational optic flow component in the optic flow 

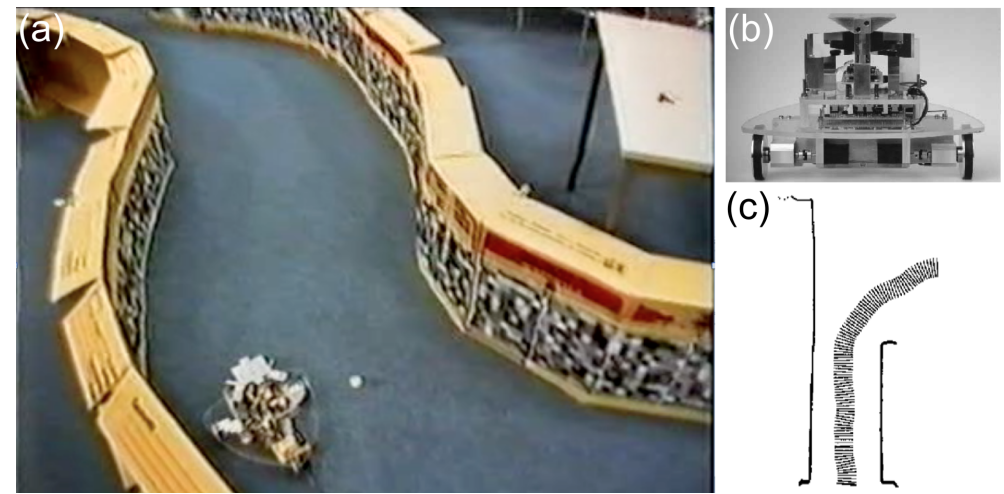

Figure 4: (a) Experimental set-up where the mobile robot built by Weber, Venkatesh, and Srinivasan (1997) tested the optic flow balance strategy in a curved corridor. (b) The robot was endowed with a camera and a couple of mirrors for looking laterally and ahead. Walls were avoided by balancing the two lateral optic flows; the robot speed was adjusted by regulating the bilateral optic flow. (c) In a straight corridor, the robot was seen to adopt a centering behavior, however, any lateral opening would make the robot leave the corridor. Adapted from [41] by permission of Oxford University Press.

measurement. Hence different techniques can be used to limit such rotational disturbances: the visual system can be counter-rotated to cancel the rotational optic flow component ([29]; [59]; [56]; [57]), the robot's rotations can be speedlimited (40]; 39]), or the rotational optic flow component can basically be subtracted from the overall optic flow by using visual cues ([42]; 446]) or by measuring mechanical rotations ([41]; [58]). An original work was developed by Dev and colleagues [42] in which only the forward speed of a wheeled robot was remotely controlled by an operator. Both distance and orientation with respect to the wall were estimated on the basis of the optic flow including the non-holomic constraint of a wheeled robot. By maintaining the lateral optic flow constant, the distance to the wall therefore became commensurate with the robot's speed given by the operator [42]. A more recent work on a holonomic and fully actuated robot was developed in simulation (60]; 37]) and implemented on-board a tiny hovercraft (61]; 62]) in which unilateral optic flow regulation was directly used to adjust the lateral distance from the walls, but the robot's heading was provided by a micro-compass (Fig 6 a). 


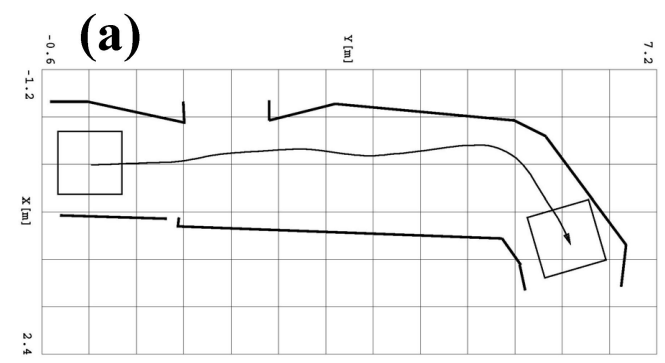

(b)

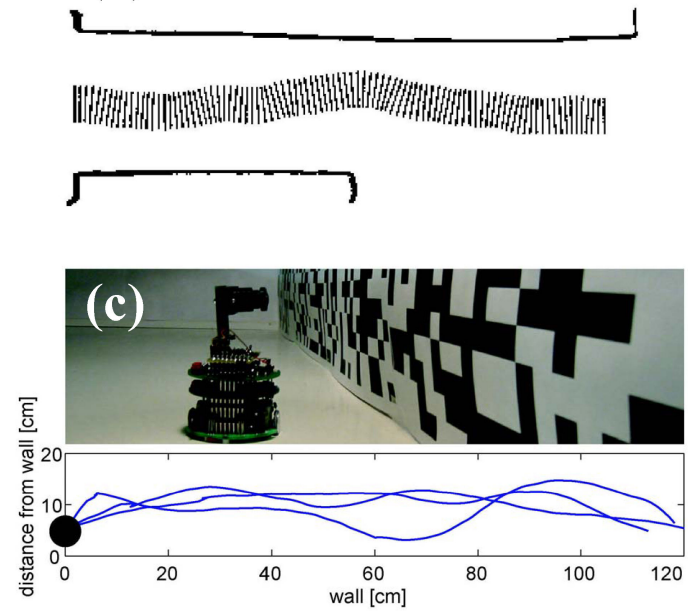

Figure 5: Wall-following behavior is obtained in mobile robotics by keeping the unilateral optic flow constant coupled with a constant forward speed. (a) Robee's behavior encountering a door-like opening from [39]. (c)Sringer. (b) Switching mode between optic-flow balance strategy to unilateral optic flow control while maintaining a constant speed from [41] by permission of Oxford University Press. (c) Wallfollowing behavior of a Khepara robot in a rectangular arena from [58]. (CIEEE.

\section{Keeping the bilateral optic flow constant: a speed control system}

The idea of introducing a speed control system based on optic flow was firstly developed by Coombs and Roberts (1992) [29]. Their Bee-Bot adjusted its forward speed to keep the optic flow within a measurable range, using a bilateral optic flow criterion to control the robot's speed. The bilateral optic flow criterion (sum of the left and right optic flows) as a feedback signal to 
directly control the robot's speed was first introduced by Santos-victor and colleagues ([38]; 39]) on-board the Robee robot. Qualitatively, the robot's speed was scaled by the level of the environment's visual clutter. In simulation, the Robot-Fly (Fig 2) moved at a constant speed in the purely translation mode during intersaccadic sequences, the robot's speed was determined by the distance from the nearest obstacle during the previous step 63. Lastly, the bilateral optic flow criterion as a feedback signal to directly control the robot's forward speed was tested on many robots in both straight and tapered corridors ([39]; 41]; 64]; 43]; 46]; 49]; 65]; 61]; 62]). The desired bilateral optic flow was $\sim 12^{\circ} / \mathrm{s}$ for the Bee-Bot robot [39, $\sim 19^{\circ} / \mathrm{s}$ in [41], $\sim 46^{\circ} / \mathrm{s}$ in [43], $\sim 21^{\circ} / \mathrm{s}$ in [46], $190^{\circ} / \mathrm{s}$ or $250^{\circ} / \mathrm{s}$ in [61]; [62]. The higher the desired bilateral optic flow, the faster the robot will be while moving close to the walls.

\section{Dual optic flow regulation}

The first optic flow regulator was originally developed for ground avoidance when following terrain ([35]; [36]). An optic flow set-point is compared to a measured optic flow to provide an error signal, this latter feeding into a regulator controlling a force orthogonal to the direction of motion. The combination of a unilateral optic flow regulator for controlling the lateral positioning on either side and a bilateral optic flow regulator for controlling the forward speed has been called a dual optic flow regulator (60]; 37$])$. The dual optic flow regulator concept was originally developed for aerial vehicles endowed with natural roll and pitch stabilization abilities, in which planar flight control systems can be developed conveniently [37] in order to mimic honeybees' abilities in the horizontal plane $([8]$; 66]; 67]). The dual optic flow regulator was for the first time simulated ([60]; 37]) then implemented on-board a 878-gram fully actuated hovercraft called LORA, which stands for Lateral Optic Regulator Autopilot (61]; 62]; Fig.77). The dual optic flow regulator is based on:

i) a unilateral optic flow regulator (Fig, 6 b) that adjusts the hovercraft's lateral thrust so as to keep the higher of the two perceived lateral optic flows (left or right) equal to a sideways optic flow set-point (noted $\left.\omega_{\text {setSide }}\right)$. The outcome is that the distance to the nearest wall y becomes proportional to the hovercaft's forward speed $V_{f}$, as determined in (ii);

ii) a bilateral optic flow regulator (Fig $6 \mathrm{c}$ ) adjusts the hovercraft's forward thrust so as to keep the sum of the two lateral optic flows (right and left) equal to a forward optic flow set-point (noted $\omega_{\text {setFwd }}$ ). 
In a steady state, with a given corridor width of $D$, the final operating point of the dual optic flow regulator will be:

$$
\begin{aligned}
& V_{f \infty}=\frac{\omega_{\text {setSide }} \cdot\left(\omega_{\text {setFwd }}-\omega_{\text {setSide }}\right)}{\omega_{\text {setFwd }}} \cdot D \\
& y_{\infty}=\frac{\omega_{\text {setFwd }}-\omega_{\text {setSide }}}{\omega_{\text {setF } w d}} \cdot D
\end{aligned}
$$

As a consequence, the robot's speed will asymptotically and automatically be scaled by the corridor width or even by the environment clutter (Fig.7b). By increasing the forward optic flow set-point $\omega_{\text {setFwd }}$ at a given sideways optic flow set-point $\omega_{\text {setSide }}$, one can change the robot's forward speed. By reducing the sideways optic flow set-point at a given forward optic flow set-point, one can induce a graceful shift from "wall-following behavior" to "centering behavior". "Centering behavior" occurs as a particular case of "wall-following behavior", whenever $\omega_{\text {setSide }} \leq \omega_{\text {setFwd }} / 2$. In addition, the dual optic flow regulator requires a third feedback loop to stabilize the robot around its vertical axis, which makes the robot experience purely translational optic flow. The robot's heading is maintained constant by a heading-lock system (based on a micro-compass enhanced by a micro-gyrometer) controlling the rear thrusters differentially in closed-loop mode (Fig 6 a).

\section{Bio-inspired visuomotor convergence}

J. Sean Humbert put forward the bio-inspired visuomotor convergence concept during his $\mathrm{PhD}(\mathrm{PhD}$ thesis [68]; obstacle avoidance and speed control [51] \& [69]; terrain-following [70]) to control a robot solely on the basis of optic flow.

This theory is based on the spatial decompositions performed by the neurons in the insect visuomotor system $([71 ; 772$; 73$])$ that extract relative velocity and proximity information from patterns of optic flow.

Based on the choice of weighting function, which is an engineering analogue to the directional templates of individual Lobula Plate Tangential Cells -LPTCneurons, various forms of relative velocity and proximity information can be obtained directly such as the lateral position and orientation [74] or the forward speed relative to corridor-like environments [65]. This resulting information can be applied as feedback to provide robust theoretically justified versions of the centering behavior (see §Optic flow balance strategy) and automatic speed adjustment behavior (see §Keeping the bilateral optic flow constant: a speed control system). Advantages of this bio-inspired approach include: 


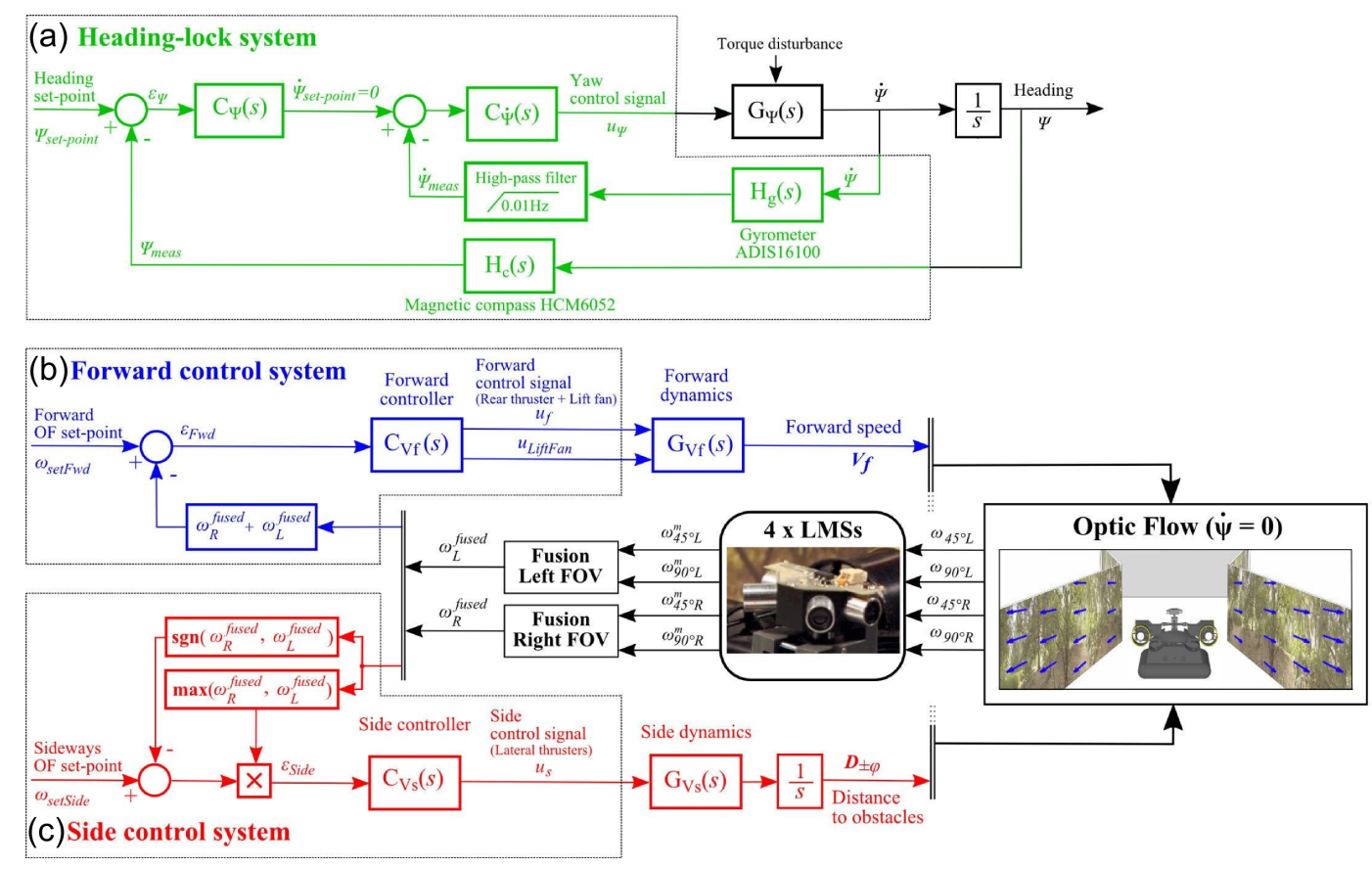

Figure 6:

i) significant improvements in signal-to-noise ratio of relative velocity and proximity information since one weight across many estimates of optic flow, an inherently noisy quantity [74],

ii) through proper choice of weighting functions, you can separate the rotational and translational components automatically and do not need to perform a separate operation [65].

The bio-inspired visuomotor convergence theory does require feedback gain selection, similar to $([37] ; 61] ; 62])$, however in $([75]$; [65]; [74]) a theoretical framework for gain selection is provided that guarantees stability of the closed loop system.

Nevertheless, it was previously demonstrated that the visual stimulation of just one fly Elementary Motion Detector (EMD) generated a strong response of the H1 LPTC-neuron, which was about $50 \%$ of its full response to thousands EMDs [76]. This kind of electrophysiological experiment has demonstrated that the feedback signal coming from optic flow is not as simple as a weighting function of each local optic flow measurement. This kind of nolinearities has not been considered by the bio-inspired visuomotor convergence theory yet. 
(a)

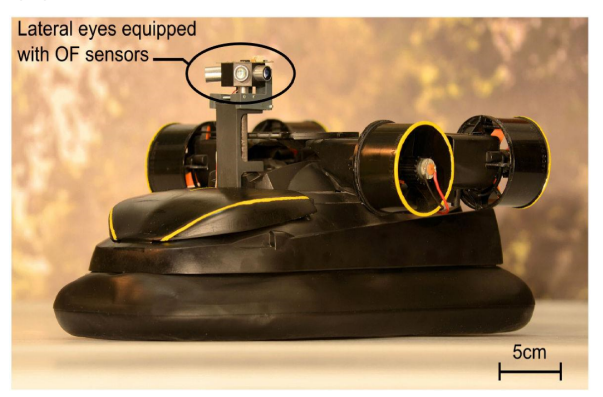

(b)

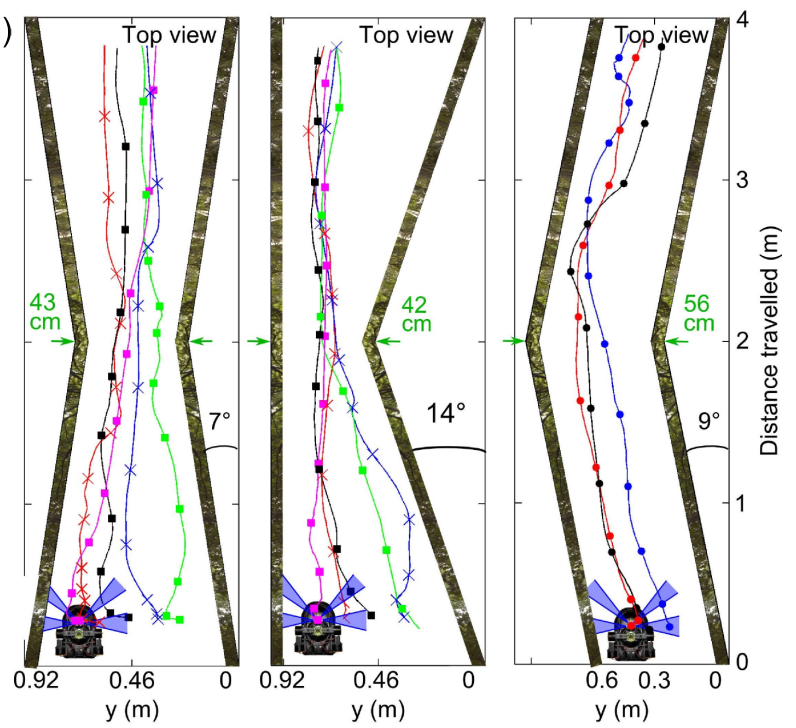

Figure 7: (a) A fully autonomous sighted hovercraft equipped with a minimalistic (8-pixel) compound eye. (b) Automatic wall-following behavior as the function of the initial ordinate in both tapered and bent corridors. From [62] under CC-BY License.

The bio-inspired visuomotor convergence was applied to a mobile robot fitted with a 1-D circular optic flow sensor providing measurements from 40 discrete angles [49]. The wheeled robot was able to move at up to $21 \mathrm{~cm} / \mathrm{s}$ by adjusting its own speed commensurate with the local corridor width (from $2 \mathrm{~m}$ to $0.8 \mathrm{~m}$ ) while following the corridor midline, even if in presence of a $45^{\circ}$-bend [49]. This same robot was able to negotiate a fixed-width $1.2 \mathrm{~m}$ corridor comprising a L-junction (65]; 77]) or in a cluttered obstacle field [77] at a fixed forward speed of $0.4 \mathrm{~m} / \mathrm{s}$ [65].

The bio-inspired visuomotor convergence was also applied to a quadrotor fitted with a 1-D circular optic flow sensor providing measurements from 20 discrete angles [75] with the help of additional metric sensors such as sonar for ground avoidance (Fig,8).

Recently, a theoretical proof for stability of the bio-inspired visuomotor convergence theory was demonstrated ([78]; 79]), but also an analysis of the robustness and quantification of the level of uncertainty in the environment (corridor-like environments with additional structure such as small poles, cylinders, or gaps and holes in the corridor) that the closed loop system can tolerate 
was provided $([78 ; 779])$. This is a major point because [78] \& [79] are the only works that have provided robustness guarantees; none of the other work listed in this review provides robustness or performance guarantees, which is a fundamental requirement for analysis of closed loop feedback systems. Actually, the only other references that provide a basic stability analysis (without robustness) of the closed loop system are references [69], [70, \& [80]. To compare the bio-inspired visuomotor convergence theory to the optic flow balance strategy that frequently fails in corridors comprising one-sided or openings in a wall (Fig. 4 \& Fig. 5). In contrast the switching mode strategy employed in such environments (Fig. 5, [41]; [39]), the bio-inspired visuomotor convergence in [78] \& [79] retains the strategy of balancing lateral optic flows and leverages the stability and performance guarantees of the closed loop to achieve stable quadrotor flight in environments that include a corridor with a large opening in a wall.
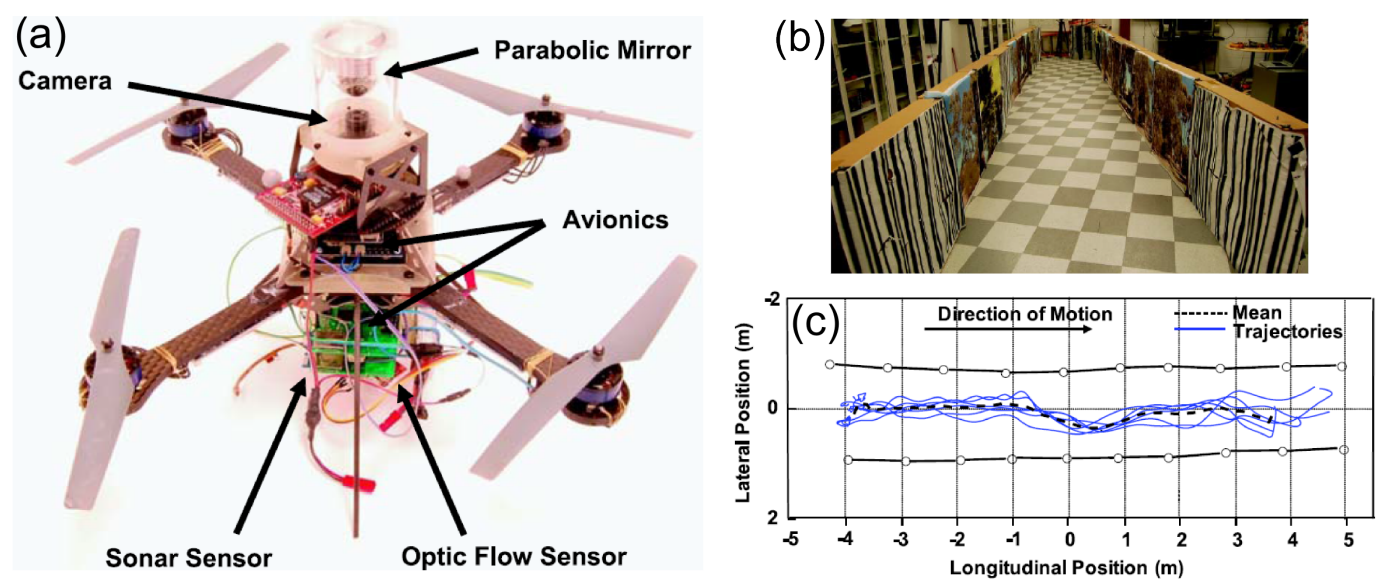

Figure 8: (a) Quadrotor with description of electronic and sensors. (b) Experimental corridor. (c) Quadrotor trajectories along the corridor. Adapted from [75]. (C)Springer.

\section{Frontal image expansion}

The optic flow balance strategy was originally suggested to explain the centering behavior along a straight corridor [8]. However, it turned out that this strategy, when used alone, did not allow an agent to avoid frontal obstacles, i.e. following a corridor that included L-junctions or T-junctions without using the frontal viewfield [40]. The frontal image expansion can therefore be 
used to estimate the time-to-contact [55] by means of the optic flow divergence ([81]; 82], and trigger a pre-specified rotation angle around the robot's vertical axis. A simulated small helicopter could therefore trigger U-turns when encountering frontal obstacles [52, or a wheeled robot could trigger a rotating angle of $90^{\circ}$ [40] or of $110^{\circ}$ [43] in front of an obstacle, or the robot could stop and rotate on the spot until the frontal range once again became large enough [41. Other robots use a series of open-loop commands, called body saccades, to avoid a frontal obstacle (Fig,9). The saccade duration has either been set to a constant pre-specified value ([83]; 84]), determined according to a Gaussian distribution [85], or modulated using optic flow ([86]; 87]; 88]; 89]; [90]). Recently, an optic-flow based algorithm has been developed to compute a quantified saccade angle; this has allowed a simulated fully actuated hovercraft to negotiate tight bends by triggering body saccades, on the basis of a time-to-contact criterion and to realign its trajectory parallel to the wall along a corridor that includes sharp turns [91].

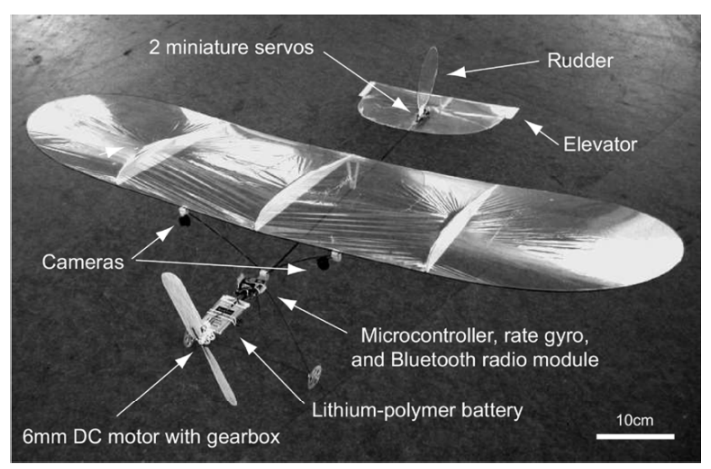

Figure 9: 30-g microflyer with description of electronic components, sensors, and actuators. Cameras on both sides sense the optic flow to assess the time-to-contact, then avoid a frontal obstacle by generating fast saccades around the microflyer's yaw axis. From [84]. (C)IEEE.

\section{OPTIC FLOW-BASED STRATEGY IN THE VERTICAL PLANE}

Ventral optic flow $\omega_{x}$ (expressed in $\mathrm{rad} \cdot \mathrm{s}^{-1}$ ) can be defined by the ratio between forward speed $V_{x}$ and flight height $z[12]$ as follows:

$$
\omega_{x}=\frac{V_{x}}{z}
$$


The ventral optic flow can be used by aerial robots to achieve different maneuvers along the longitudinal axis: take off, terrain-following, flying nap-of-the earth, landing, decking. 20 years ago, a landing strategy was put forward to explain how a bee could land on flat ground ([66]; 92]). Bees were observed to land on flat ground with a constant descent angle, M.V. Srinivasan and colleagues at the Australian National University therefore suggested a pair of rules for explaining the bee's smooth landing on flat ground: (i) the forward speed is controlled by holding the angular velocity of the image of the ground constant (i.e., holding a constant ventral optic flow), (ii) making the instantaneous downward speed proportional to the instantaneous forward speed (i.e., holding a constant descent angle). This pair of rules was first implemented in a robotic gantry without including dynamic aspects [92, and subsequently on-board a small fixed-wing aircraft 93 in which the elevator angle was controlled via a proportional feedback loop by holding the ventral optic flow close to $\sim 40^{\circ} / \mathrm{s}$. During a real closed-loop flight experiment, the small fixed-wing aircraft was seen to slow down from $25 \mathrm{~m} / \mathrm{s}$ to $15 \mathrm{~m} / \mathrm{s}$ while loosing $30 \mathrm{~m}$ in altitude, but experimental results were almost the same with or without the feedback loop, and the craft's altitude was observed to decrease linearly with time instead of exponentially as predicted by the pair of rules [93]. Ventral optic flow was also employed for ground avoidance on-board a Micro Air Vehicle (MAV) whose mass was lower than 100-gram. A control algorithm based on a "bang-bang" method was used on-board a MAV to control its lift such that if a certain threshold of ventral optic flow was exceeded, the MAV elevator angle would be moved to a preset deflection (glider in [87]; fixed-wing aircraft in [88]). In 2002, a 840-gram tethered rotorcraft, called Fania, was able to jump over 1-meter high obstacles using a basic optic-flow based control algorithm 94.

A small Hirobo Eagle helicopter with an overall mass of $8 \mathrm{~kg} 32$ used its avionics to deduce the height above ground from the ventral optic flow. The above ground height was therefore held constant by adjusting the main rotor thrust: the helicopter was able to follow the terrain at a flight height of $\sim 1.5-2 \mathrm{~m}$ while flying at speeds ranging from 5 to $8 \mathrm{~m} / \mathrm{s}$ (Fig.11).

\section{Ventral optic flow regulation}

In 2003, a 100-gram tethered rotorcraft, called OCTAVE (OCTAVE stands for Optic flow based Control system for Aerial VEhicles), followed a smooth relief [95] by using the ventral optic flow regulator principle ([35], Fig, 10p). A ventral optic flow regulator integrated an optic flow measurement into a feedback loop driving the robot's lift so as to compensate for any deviations in the measured optic flow from a given optic flow set-point (The OCTAVE autopilot Fig 10). 
The ventral optic flow regulator ensures that at any moment the flight height is proportional to the airspeed. Any increase in the airspeed leads to an increase in height, so this can lead to an automatic takeoff then terrain-following under visual control. In the same way, any decrease in the airspeed can lead to an automatic landing at a constant angle under visual control until touchdown at zero speed [36], as actually observed in honeybees in similar situations ([66; 92]). The OCTAVE robot was also able to land along the longitudinal axis onto a moving platform [96, as honeybees actually do on a moving target [97].

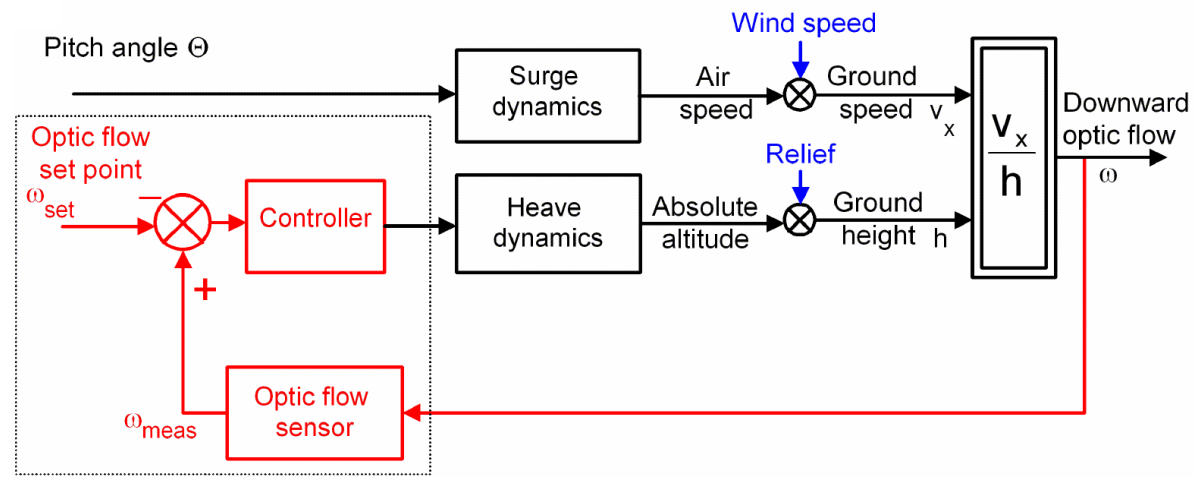

Figure 10: OCTAVE autopilot based on a ventral optic flow regulator (OCTAVE stands for Optic flow based Control system for Aerial VEhicles). The optic flow regulator controls the lift at all times so as to maintain the downward optic flow constant despite wind and relief disturbances. From [36]. (C)Elsevier.

A Vertical Take-Off and Landing (VTOL)aircraft with an overall mass of $0.85 \mathrm{~kg}$ [98] was able to follow a steep terrain (slope 25\%) at a flight height of $1.5 \mathrm{~m}$ while flying at $0.3 \mathrm{~m} / \mathrm{s}$ by regulating the ventral optic flow at $\sim 12^{\circ} / \mathrm{s}$ while estimating its own forward speed by combining both accelerometer and barometer readings [8].

\section{Twin dual optic flow regulation}

With the goal of developing a full optic flow-based autopilot for 3D indoor navigation, the OCTAVE autopilot for ground avoidance [35] and LORA autopilot for speed control and lateral obstacle avoidance [37] have been combined to develop the ALIS autopilot (ALIS stands for Autopilot using an Insect-based vision System) [99]. The ALIS autopilot consists of two visuo-motor feedback loops: (i) the speed control loop (along x-axis) based on a feedback signal 

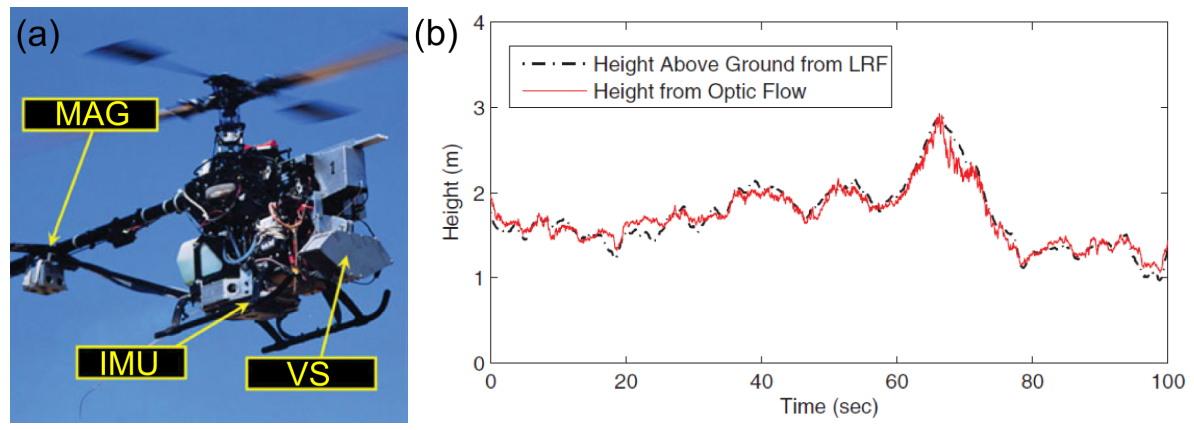

Figure 11: (a) 8-kg Hirobo Eagle helicopter. (b) Helicopter height measured by a Laser Range Finder (LRF) versus the height estimated from both optic flow and forward velocity derived from GPS. MAG stands for MAGnetometer; IMU stands for Inertial Measurement Unit; VS stands for Vision Sensor. From [32] reprinted by permission of John Wiley \& Sons, Inc.

coming from the maximum value of the bi-lateral or bi-vertical optic flow; consequently the agent considers the minimum cross-section of the tunnel when adjusting its forward speed, and (ii) a positioning control loop (along y- and z-axis) based on a feedback signal coming from the maximum value of the lateral, ventral, or dorsal optic flow; consequently the distance from the nearest surface (lateral walls, ground, or ceiling) becomes proportional to the forward speed obtained in (i). These two loops work in parallel and are independent; each has its own optic flow set-point. Simulation results 99] showed that an agent was able to navigate safely along a straight or tapered tunnel and to react appropriately to any untoward optic flow perturbations, such as those resulting from the occasional lack of texture on one wall or converging-diverging tunnel sections.

Recently, dual flow optic regulation in the vertical plane was tested on-board a 80-gram rotorcraft called BeeRotor [100]. As a third control feedback, an active system of reorientation based on a quasi-panoramic eye constantly realigned its gaze in parallel to the nearest surface followed: the Beerotor robot demonstrated its abilities and achieved automatic terrain following despite steep reliefs (Fig 12) without a need for inertial frames or scaling sensors [100].

Recently, in the framework of the Green Brain project managed by James Marshall, a dual optic flow regulator for both speed control and lateral positioning, and a ventral optic flow for altitude control were implemented on-board a small quadrotor ([101]). 


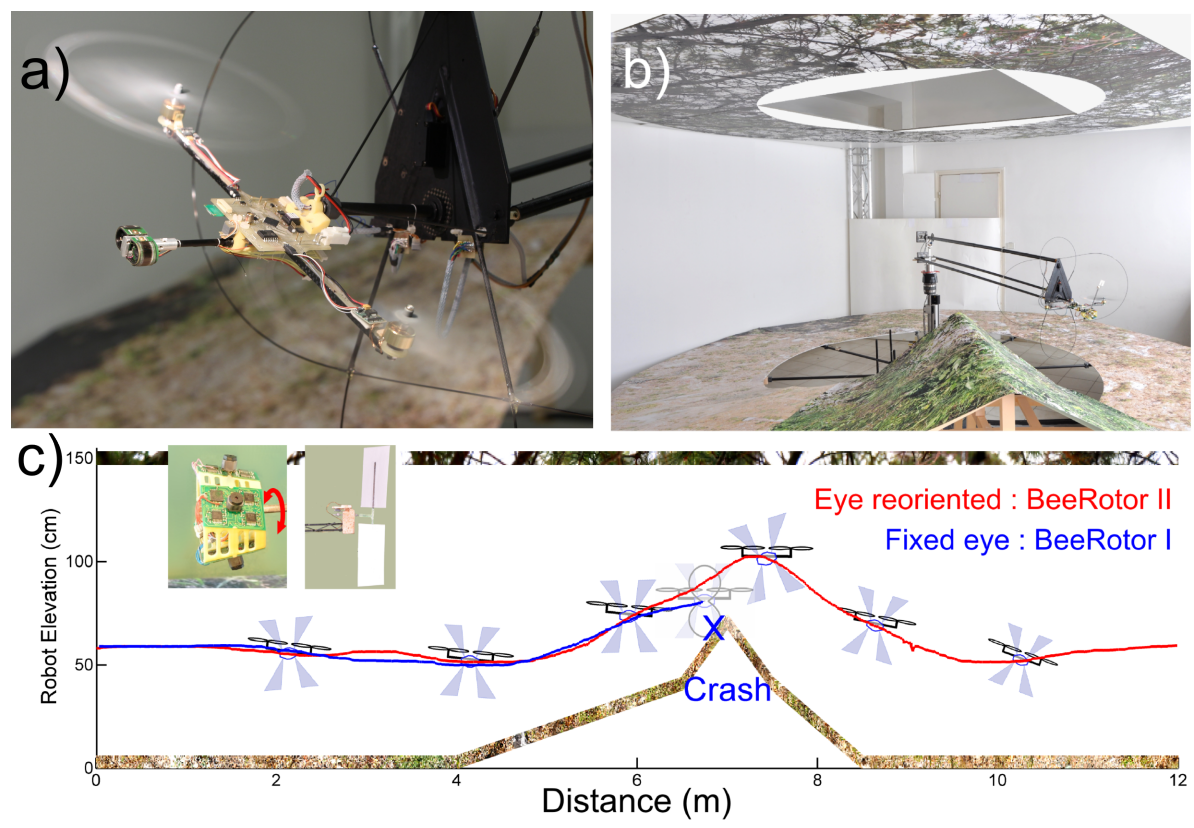

Figure 12: (a) BeeRotor I robot equipped with the full cylindrical CurACE sensor [18]. (b) Photograph of the experimental set-up. (c) Trajectories of the robot BeeRotor II that automatically follows the ground, thanks to the ventral optic flow regulator and the fixed eye (blue) and decoupled eye (red) oriented parallel to the ground. This reorientation enables the robot, at an earlier stage, to detect the increase in the optic flow due to steep relief. Adapted from [100] under CC-BY license.

\section{Ventral image expansion}

The expansion of the ventral optic flow can be used for VTOL aircraft. This kind of aircraft is able to take off, hover, and land vertically. If the robot is looking straight down, the ventral image expansion can be computed by the optic flow field divergence $\nabla \vec{\omega}$ (expressed in $s^{-1}$ ), which is equal to:

$$
\nabla \vec{\omega}=\omega_{z}=-\frac{V_{z}}{z}
$$

with $z$ the height above the ground, and $V_{z}$ the ascent speed (axis pointing up). This optic flow divergence can also be expressed in terms of time-tocontact $\tau$ (expressed in $s$ ) [55]: 


$$
\tau=\frac{1}{\omega_{z}}=-\frac{z}{V_{z}}
$$

The ventral image expansion by means of optic flow divergence or time-tocontact allows a VTOL vehicle fitted with a monocular camera and an inertial measurement unit to take off, hover or land vertically without measurement of flight height or vertical speed. Methods using either an enforcement of a decreasing time-to-contact or keeping the optic flow divergence constant have been used in recent landings ([80]; 102]; 103]; [104]; 105]), as actually observed in honeybees in similar situations [106]. Automatic vertical landing can be achieved using vertical optic flow (the downwards expansion of optic flow) even over a moving platform ([80]; [107]), this kind of maneuver is called a decklanding and could be useful for any VTOL aircraft wanting to land on the deck of a sea going vessel (Fig,13).

\section{OPTIC FLOW-BASED ODOMETRY}

There is now evidence that bees gauge distance in flight by integrating optic flow over time [108]. Lateral optic flows were used to gauge the traveled distance of wheeled robots ([41]; 45]) or a small blimp [50]. Downward optic flow was used to gauge the traveled distance of a car-like robot [109] or a wheeled robot [110. An optic-flow based odometer appears to achieve better results than a wheelbased odometer [109]. A recent paper demonstrated that the combination of downward optic flow and stereovision to calculate the current position of a quadrotor along a pre-defined trajectory could be achieved with an error of about $1.7 \%$ in the total path length [11].

\section{RECENT ADVANCES ON OPTIC FLOW-BASED ROBOTS}

Recently, an event-based collision avoidance algorithm was developed and has been implemented on-board a wheeled robot [112. The collision avoidance direction, computed from this algorithm, points away from nearby objects by combining optic flow measurement and true ground speed measurement. This new kind of bio-inspired algorithm has only been tested under open loop conditions in a corridor with a small number of objects but experimental results have shown that this algorithm could be used to control a robotic platform 112. 
(a)
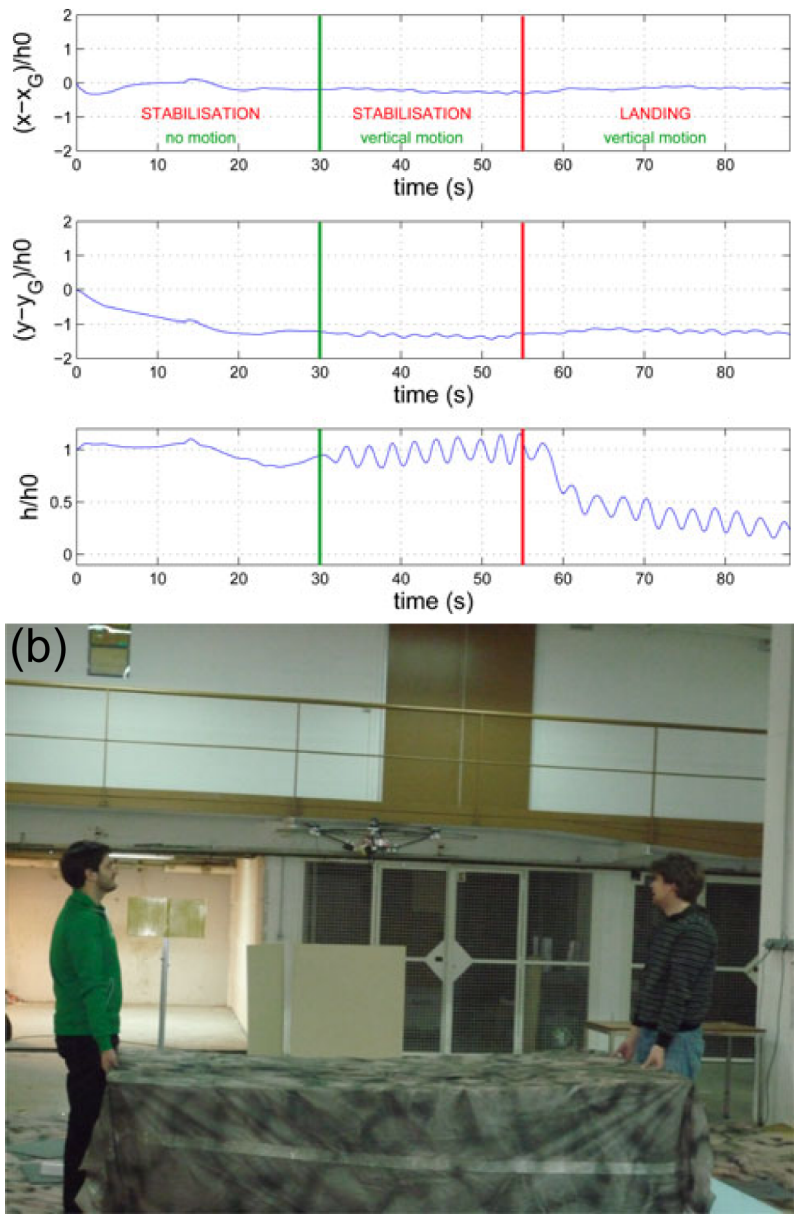

Figure 13: (a) Quadrotor position during three experiments. Despite the surface below moving with small oscillations, the quadrotor followed the surface oscillations either at a constant height above the surface or during a vertical landing maneuver. (b) Quadrotor fitted with a downward looking camera above an oscillating surface. From [80]. (C)IEEE.

A new approach to solving the "Route Learning and Long-range Homing" problem has been suggested in recent years: Denuelle and colleagues have combined a smart optic flow method and a snap-shot panoramic picture to navigate back and forth between the home and the goal locations [113. 
Efforts have also been made in recent years to decrease drift due to low mass inertial devices on-board robots by using optic flow direction [114]: optic flow direction can be defined by the direction along the focus of the expansion and contraction axes.

Finally, a new kind of robust dedicated sensor for the measurement of changes over extended periods -several decades - of light level has also been recently developed to measure optic flow even in the dark ([18]; [115]).

\section{COMMERCIAL DEVELOPMENT}

Several companies are now using optic flow onboard aerial robots, examples of this are the Parrot company with the AR Drone to stabilize the $\mathrm{x}-\mathrm{y}$ position in hover mode or Sensefly with the fixed-wing drone called eBee that lands automatically. It is worth noting that the SnapDragon Flight controller from Qualcomm now features an optic flow sensor as part of its avionics.

\section{Acknowledgements}

We thank D. Wood for improving the English manuscript.

\section{References}

[1] G. K. Taylor and H. G. Krapp, "Sensory systems and flight stability: what do insects measure and why?" Advances in insect physiology, vol. 34, pp. 231-316, 2007.

[2] H. G. Krapp, R. Hengstenberg et al., "Estimation of self-motion by optic flow processing in single visual interneurons," Nature, vol. 384, no. 6608, pp. 463-466, 1996.

[3] N. Franceschini, F. Ruffier, J. Serres, and S. Viollet, Optic flow based visual guidance: from flying insects to miniature aerial vehicles. INTECH Open Access Publisher, 2009.

[4] M. V. Srinivasan, "Honeybees as a model for the study of visually guided flight, navigation, and biologically inspired robotics," Physiological Reviews, vol. 91, no. 2, pp. 413-460, 2011.

[5] N. Franceschini, "Small brains, smart machines: from fly vision to robot vision and back again," Proceedings of the IEEE, vol. 102, no. 5, pp. 751-781, 2014. 
[6] C. T. David, "Compensation for height in the control of groundspeed bydrosophila in a new, 'barber's pole'wind tunnel," Journal of Comparative Physiology A: Neuroethology, Sensory, Neural, and Behavioral Physiology, vol. 147, no. 4, pp. 485-493, 1982.

[7] W. Kirchner and M. Srinivasan, "Freely moving honeybees use image motion to estimate distance," Naturwissenchaften, vol. 76, pp. 281-282, 1989.

[8] M. Srinivasan, M. Lehrer, W. Kirchner, and S. Zhang, "Range perception through apparent image speed in freely flying honeybees," Visual neuroscience, vol. 6, no. 05, pp. 519-535, 1991.

[9] E. Baird, M. V. Srinivasan, S. Zhang, and A. Cowling, "Visual control of flight speed in honeybees," Journal of Experimental Biology, vol. 208, no. 20, pp. 3895-3905, 2005.

[10] M. Ibbotson, "Evidence for velocity-tuned motion-sensitive descending neurons in the honeybee," Proceedings of the Royal Society of London B: Biological Sciences, vol. 268, no. 1482, pp. 2195-2201, 2001.

[11] J. Gibson, The perception of the visual world. Boston: Houghton Mifflin, 1950.

[12] T. C. Whiteside and G. Samuel, "Blur zone," Nature, vol. 225, pp. 94-95, 1970.

[13] K. Nakayama and J. Loomis, "Optical velocity patterns, velocity-sensitive neurons, and space perception: a hypothesis," Perception, vol. 3, no. 1, pp. 63-80, 1974.

[14] J. J. Koenderink, "Optic flow," Vision research, vol. 26, no. 1, pp. 161$179,1986$.

[15] J. J. Koenderink and A. J. van Doorn, "Facts on optic flow," Biological cybernetics, vol. 56, no. 4, pp. 247-254, 1987.

[16] E. Buchner, Photoreception and vision in invertebrates. NATO ASI Series, Series A: Life Science, vol. 74, Plenum, 1984, ch. Behavioural analysis of spatial vision in insects, pp. 561-621.

[17] R. Moeckel and S.-C. Liu, "Motion detection chips for robotic platforms," in Flying Insects and Robots. Springer Berlin Heidelberg, 2009, pp. 101114 . 
[18] D. Floreano, R. Pericet-Camara, S. Viollet, F. Ruffier, A. Brückner, R. Leitel, W. Buss, M. Menouni, F. Expert, R. Juston et al., "Miniature curved artificial compound eyes," Proceedings of the National Academy of Sciences, vol. 110, no. 23, pp. 9267-9272, 2013.

[19] H. Chao, Y. Gu, and M. Napolitano, "A survey of optical flow techniques for robotics navigation applications," Journal of Intelligent $\&$ Robotic Systems, vol. 73, no. 1-4, pp. 361-372, 2014.

[20] D. Lucas and T. Kanade, "An iterative image registration technique with an application to stereo vision," in In proceedings of the 7th International Joint Conference on Artificial Intelligence (IJCAI), (pp. 674-679), Vancouver, BC., 1981.

[21] B. K. P. Horn and B. Schunck, "Determining optical flow," Artificial Intelligence, vol. 17, pp. 185-203, 1981.

[22] C. Blanes, Appareil visuel elementaire pour la navigation a vue d'un robot mobile autonome. Marseille: Master thesis in Neurosciences (DEA in French), Neurosciences, Univ. Aix-Marseille II, 1986.

[23] J.-M. Pichon, C. Blanes, and N. Franceschini, "Visual guidance of a mobile robot equipped with a network of self-motion sensors," in Proc. of SPIE Conf. on Mobile Robots IV, W.J. Wolfe and W.H. Chun, Eds. Bellingham, U.S.A,: SPIE Vol. 1195, 1989, pp. 44-53.

[24] N. Franceschini, J. M. Pichon, and B. C, "From insect vision to robot vision," Philosophical Transaction: Biological Sciences, vol. 337, pp. 283294, 1992.

[25] M. F. Land and T. Collett, "Chasing behaviour of houseflies (fannia canicularis)," Journal of Comparative Physiology, vol. 89, no. 4, pp. 331-357, 1974 .

[26] C. Schilstra and J. Hateren, "Blowfly flight and optic flow. i. thorax kinematics and flight dynamics," Journal of Experimental Biology, vol. 202, no. 11, pp. 1481-1490, 1999.

[27] L. F. Tammero and M. H. Dickinson, "The influence of visual landscape on the free flight behavior of the fruit fly drosophila melanogaster," Journal of Experimental Biology, vol. 205, no. 3, pp. 327-343, 2002. 
[28] R. Kern, N. Boeddeker, L. Dittmar, and M. Egelhaaf, "Blowfly flight characteristics are shaped by environmental features and controlled by optic flow information," The Journal of experimental biology, vol. 215, no. 14, pp. 2501-2514, 2012.

[29] D. Coombs and K. Roberts, "Bee-bot: using peripheral optical flow to avoid obstacles," in In SPIE: Vol. 1825. Intelligent robots and computer vision XI, pp. 714-721, 1992.

[30] — - "Centering behavior using peripheral vision," in Computer Vision and Pattern Recognition, 1993. Proceedings CVPR'93., 1993 IEEE Computer Society Conference on. IEEE, 1993, pp. 440-445.

[31] S. Griffiths, J. Saunders, A. Curtis, B. Barber, T. McLain, and R. Beard, "Obstacle and terrain avoidance for miniature aerial vehicles," in $A d$ vances in Unmanned Aerial Vehicles. Springer, 2007, pp. 213-244.

[32] M. A. Garratt and J. S. Chahl, "Vision-based terrain following for an unmanned rotorcraft," Journal of Field Robotics, vol. 25(4-5), pp. 284$301,2008$.

[33] A. Beyeler, J.-C. Zufferey, and D. Floreano, "Vision-based control of nearobstacle flight," Autonomous robots, vol. 27, no. 3, pp. 201-219, 2009.

[34] F. Mura and N. Franceschini, "Visual control of altitude and speed in a flying agent," in From Animals to Animats III, D. Cliff et al., Eds. Cambridge, U.S.A: MIT Press, 1994, pp. 91-99.

[35] F. Ruffier and N. Franceschini, "Optic flow regulation: the key to aircraft automatic guidance," Robotics and Autonomous Systems, vol. 50, no. 4, pp. 177-194, 2005.

[36] N. Franceschini, F. Ruffier, and J. Serres, "A bio-inspired flying robot sheds light on insect piloting abilities," Current Biology, vol. 17(4), pp. 329-335, 2007.

[37] J. Serres, D. Dray, F. Ruffier, and N. Franceschini, "A vision-based autopilot for a miniature air vehicle: joint speed control and lateral obstacle avoidance," Autonomous Robots, vol. 25, no. 1-2, pp. 103-122, 2008.

[38] J. Santos-Victor, G. Sandini, F. Curotto, and S. Garibaldi, "Divergent stereo for robot navigation: Learning from bees," in Computer Vision and Pattern Recognition, 1993. Proceedings CVPR'93., 1993 IEEE Computer Society Conference on. IEEE, 1993, pp. 434-439. 
[39] —_ "Divergent stereo in autonomous navigation: From bees to robots," International Journal of Computer Vision, vol. 14, no. 2, pp. 159-177, 1995.

[40] A. P. Duchon and W. H. Warren, "Robot navigation from a gibsonian viewpoint," in Systems, Man, and Cybernetics, 1994. Humans, Information and Technology., 1994 IEEE International Conference on, vol. 3. IEEE, 1994, pp. 2272-2277.

[41] V. S. Weber, K. and M. Srinivasan, From Living eyes to Seeing Machines. Oxford University Press, Oxford, UK, 1997, no. 11, ch. Insect inspired behaviors for the autonomous robots, pp. 226-248.

[42] A. Dev, B. Krose, and F. Groen, "Navigation of a mobile robot on a temporal development of the optic flow," in In proceedings of the IEEE/RSJ International Conference on Intelligent Robots and Systems (IROS), pp. 558-563, Grenoble, France, 1997.

[43] G. Baratoff, C. Toepfer, and H. Neumann, "Combined space-variant maps for optical flow navigation," Biological Cybernetics, vol. 83(3), pp. 199$209,2000$.

[44] R. Carelli, C. Soria, O. Nasisi, and E. Freire, "Stable agv corridor navigation with fused vision-based controls signals," in In proceedings the 28th IEEE Conference of Industrial Electronics Society (IECON), vol. 3, pp. 2433-2438, Sevilla, Spain., 2002.

[45] C. McCarthy and N. Barnes, "Performance of temporal filters for optical flow estimation in mobile robot corridor centring and visual odometry," in Proceedings of the 2003 Australasian Conference on Robotics 85 Automation, 2003.

[46] A. Argyros, D. Tsakiris, and C. Groyer, "Biomimetic centering behavior for mobile robots with panoramic sensors," IEEE Robotics and Automation Magazine, Special issue on "Mobile robots with panoramic sensors", K. Daniilides and N. Papakolopoulos (Eds.), vol. 11, pp. 21-30, 2004.

[47] S. Hrabar, G. Sukatme, P. Corke, K. Usher, and J. Roberts, "Combined optic-flow and stereo-based navigation of urban canyons for a uav," in In proceedings of the IEEE/RSJ International Conference on Intellignent Robots and Systems (IROS), pp. 3309-3316, Edmonton, Alberta, Canada, 2005. 
[48] K. Souhila and A. Karim, "Optical flow based robot obstacle avoidance," International Journal of Advanced Robotic Systems, vol. 4, no. 1, pp. 13-16, 2007.

[49] J. S. Humbert, A. Hyslop, and M. Chinn, "Experimental validation of wide-field integration methods for autonomous navigation," in Intelligent Robots and Systems, 200\%. IROS 200\%. IEEE/RSJ International Conference on. IEEE, 2007, pp. 2144-2149.

[50] F. Iida, "Goal-directed navigation of an autonomous flying robot using biogically inspired cheap vision," in Proceedings of the 32nd International Symposium on Robotics (ISR), pp. 1404-1409, 2001.

[51] J. S. Humbert, R. M. Murray, and M. H. Dickinson, "Sensorimotor convergence in visual navigation and flight control systems," in Proceedings of the 16th IFAC World Congress. Praha, Czech Republic, 2005.

[52] L. Muratet, S. Doncieux, Y. Briere, and J. Meyer, "A contribution to vision-based autonomous helicopter flight in urban environments," Robotics and Autonomous Systems, vol. 50(4), pp. 195-209, 2005.

[53] S. Griffiths, J. Saunders, A. Curtis, B. Barber, T. McLain, and R. Beard, "Maximizing miniature aerial vehicles," Robotics \& Automation Magazine, IEEE, vol. 13, no. 3, pp. 34-43, 2006.

[54] S. Hrabar and G. Sukatme, "Optimum camera angle for optic flow-based centring response," in In proceedings of the IEEE International Conference on Robotics and Automation (ICRA), pp. 3922-3927, Beijing, China., 2006.

[55] D. N. Lee, "A theory of visual control of braking based on information about time-to-collision," Perception, vol. 5, no. 4, pp. 437-459, 1976.

[56] J. Serres, F. Ruffier, and N. Franceschini, "Biomimetic visual navigation in a corridor: to centre or not to centre," in Proceedings of International Mediterranean Modeling Multiconference. I3M'05, 2005, pp. 91-97.

[57] J. Serres, F. Ruffier, S. Viollet, and N. Franceschini, "Toward optic flow regulation for wall-following and centring behaviours," International Journal of Advanced Robotic Systems, vol. 3, no. 2, pp. 147-154, 2006.

[58] J.-C. Zufferey and D. Floreano, "Toward 30-gram autonomous indoor aircraft: Vision-based obstacle avoidance and altitude control," in Robotics 
and Automation, 2005. ICRA 2005. Proceedings of the 2005 IEEE International Conference on. IEEE, 2005, pp. 2594-2599.

[59] M. A. Lewis, "Visual navigation in a robot using zig-zag behavior." in NIPS. Citeseer, 1997, pp. 822-828.

[60] J. Serres, F. Ruffier, and N. Franceschini, "Two optic flow regulators for speed control and obstacle avoidance," in Biomedical Robotics and Biomechatronics, 2006. BioRob 2006. The First IEEE/RAS-EMBS International Conference on. IEEE, 2006, pp. 750-757.

[61] F. L. Roubieu, J. Serres, N. Franceschini, F. Ruffier, and S. Viollet, "A fully-autonomous hovercraft inspired by bees: wall following and speed control in straight and tapered corridors," in Robotics and Biomimetics (ROBIO), 2012 IEEE International Conference on. IEEE, 2012, pp. $1311-1318$.

[62] F. L. Roubieu, J. R. Serres, F. Colonnier, N. Franceschini, S. Viollet, and F. Ruffier, "A biomimetic vision-based hovercraft accounts for bees' complex behaviour in various corridors," Bioinspiration $\mathcal{E}$ biomimetics, vol. 9, no. 3, p. $036003,2014$.

[63] N. Martin and N. Franceschini, "Obstacle avoidance and speed control in a mobile vehicle equipped with a compound eye," in Intelligent Vehicles' 94 Symposium, Proceedings of the. IEEE, 1994, pp. 381-386.

[64] M. V. Srinivasan, J. S. Chahl, K. Weber, S. Venkatesh, M. G. Nagle, and S.-W. Zhang, "Robot navigation inspired by principles of insect vision," Robotics and Autonomous Systems, vol. 26, no. 2, pp. 203-216, 1999.

[65] J. S. Humbert and A. M. Hyslop, "Bioinspired visuomotor convergence," Robotics, IEEE Transactions on, vol. 26, no. 1, pp. 121-130, 2010.

[66] M. Srinivasan, S. Zhang, M. Lehrer, and T. Collett, "Honeybee navigation en route to the goal: visual flight control and odometry," The Journal of Experimental Biology, vol. 199, no. 1, pp. 237-244, 1996.

[67] J. Serres, M. G, F. Ruffier, and N. Franceschini, "A bee in the corridor: centering and wall-following," Naturwissenschaften, vol. 95, pp. 11811187, 2008.

[68] J. S. Humbert, "Bio-inspired visuomotor convergence in navigation and flight control systems," Ph.D. dissertation, California Institute of Technology, 2005. 
[69] J. S. Humbert, R. M. Murray, and M. H. Dickinson, "A control-oriented analysis of bio-inspired visuomotor convergence," in Decision and Control, 2005 and 2005 European Control Conference. CDC-ECC'05. 44th IEEE Conference on. IEEE, 2005, pp. 245-250.

[70] — , "Pitch-altitude control and terrain following based on bio-inspired visuomotor convergence," in AIAA Conference on Guidance, Navigation and Control, 2005.

[71] K. Hausen, "Motion sensitive interneurons in the optomotor system of the fly," Biological Cybernetics, vol. 45, no. 2, pp. 143-156, 1982.

[72] H. G. Krapp, B. Hengstenberg, and R. Hengstenberg, "Dendritic structure and receptive-field organization of optic flow processing interneurons in the fly," Journal of Neurophysiology, vol. 79, no. 4, pp. 1902-1917, 1998.

[73] A. Borst and J. Haag, "Neural networks in the cockpit of the fly," Journal of Comparative Physiology A, vol. 188, no. 6, pp. 419-437, 2002.

[74] A. Hyslop, H. G. Krapp, and J. S. Humbert, "Control theoretic interpretation of directional motion preferences in optic flow processing interneurons," Biological cybernetics, vol. 103, no. 5, pp. 353-364, 2010.

[75] J. Conroy, G. Gremillion, B. Ranganathan, and J. S. Humbert, "Implementation of wide-field integration of optic flow for autonomous quadrotor navigation," Autonomous robots, vol. 27, no. 3, pp. 189-198, 2009.

[76] N. Franceschini, A. Riehle, and A. Le Nestour, "Directionally selective motion detection by insect neurons," in Facets of vision. Springer, 1989, pp. 360-390.

[77] A. Hyslop, H. G. Krapp, and J. S. Humbert, "Control theoretic interpretation of directional motion preferences in optic flow processing interneurons," Biological cybernetics, vol. 103, no. 5, pp. 353-364, 2010.

[78] J. Keshavan, G. Gremillion, H. Escobar-Alvarez, and J. Humbert, "A $\mu$ analysis-based, controller-synthesis framework for robust bioinspired visual navigation in less-structured environments," Bioinspiration $\&$ biomimetics, vol. 9, no. 2, p. 025011, 2014.

[79] J. Keshavan, G. Gremillion, H. Alvarez-Escobar, and J. S. Humbert, "Autonomous vision-based navigation of a quadrotor in corridor-like environments," International Journal of Micro Air Vehicles, vol. 7, no. 2, pp. 111-124, 2015. 
[80] B. Herissé, T. Hamel, R. Mahony, and F.-X. Russotto, "Landing a vtol unmanned aerial vehicle on a moving platform using optical flow," Robotics, IEEE Transactions on, vol. 28, no. 1, pp. 77-89, 2012.

[81] R. Nelson and J. Aloimonos, "Using flow field divergence for obstacle avoidance in visual navigation," in Science Applications International Corp, Proceedings: Image Understanding Workshop,, vol. 2, 1988.

[82] N. Ancona and T. Poggio, "Optical flow from 1d correlation: Application to a simple time-to-crash detector," in 4th International Conference on Computer Vision, Proceedings of the, Berlin, Germany, 1993, pp. 209214.

[83] S. B. i Badia, U. Bernardet, and P. F. Verschure, "Non-linear neuronal responses as an emergent property of afferent networks: a case study of the locust lobula giant movement detector," PLoS Comput Biol, vol. 6, no. 3, p. e1000701, 2010.

[84] J.-C. Zufferey and D. Floreano, "Fly-inspired visual steering of an ultralight indoor aircraft," Robotics, IEEE Transactions on, vol. 22, no. 1, pp. 137-146, 2006.

[85] M. B. Reiser and M. H. Dickinson, "A test bed for insect-inspired robotic control," Philosophical Transactions of the Royal Society of London A: Mathematical, Physical and Engineering Sciences, vol. 361, no. 1811, pp. 2267-2285, 2003.

[86] A. Beyeler, J.-C. Zufferey, and D. Floreano, "3d vision-based navigation for indoor microflyers," in Robotics and Automation, 2007 IEEE International Conference on. IEEE, 2007, pp. 1336-1341.

[87] G. Barrows, C. Neely, and K. Miller, "Optic flow sensors for mav navigation," in Fixed and flapping wing aerodynamics for Micro Air Vehicle applications. Bellingham, U.S.A.: Progress in Astronautics and Aeronautics, AIAA, Vol. 195, 2001, pp. 557-574.

[88] W. E. Green, P. Y. Oh, and G. Barrows, "Flying insect inspired vision for autonomous aerial robot maneuvers in near-earth environments," in Robotics and Automation, 2004. Proceedings. ICRA'04. 2004 IEEE International Conference on, vol. 3. IEEE, 2004, pp. 2347-2352.

[89] J. P. Lindemann, H. Weiss, R. Möller, and M. Egelhaaf, "Saccadic flight strategy facilitates collision avoidance: closed-loop performance of a cyberfly," Biological cybernetics, vol. 98, no. 3, pp. 213-227, 2008. 
[90] M. Rezaei and F. Saghafi, "Optical flow-based obstacle avoidance of a fixed-wing mav," Aircraft Engineering and Aerospace Technology, vol. 83, no. 2, pp. 85-93, 2011.

[91] J. R. Serres and F. Ruffier, "Biomimetic autopilot based on minimalistic motion vision for navigating along corridors comprising u-shaped and sshaped turns," Journal of Bionic Engineering, vol. 12, no. 1, pp. 47-60, 2015 .

[92] M. Srinivasan, S. Zhang, J. Chahl, E. Barth, and S. Venkatesh, "How honeybees make grazing landings on flat surfaces," Biological Cybernetics, vol. 83(3), pp. 171-183, 2000.

[93] J. Chahl, M. Srinivasan, and S. Zhang, "Landing strategies in honeybees and applications to uninhabited airborne vehicles," Int. J. of Robotics Research, vol. 23(2), pp. 101-110, 2004.

[94] T. Netter and N. Franceschini, "A robotic aircraft that follows terrain using a neuromorphic eye," in Proc. of IEEE Conf. on Intelligent Robots and Systems (IROS). Lausanne, Switzerland: Progress in Astronautics and Aeronautics, AIAA, Vol. 195, 2002, pp. 129-134.

[95] F. Ruffier, S. Viollet, S. Amic, and N. Franceschini, "Bio-inspired optical flow circuits for the visual guidance of micro air vehicles," in Circuits and Systems, 2003. ISCAS'03. Proceedings of the 2003 International Symposium on, vol. 3. IEEE, 2003, pp. III-846.

[96] F. Ruffier and N. Franceschini, "Optic flow regulation in unsteady environments: a tethered mav achieves terrain following and targeted landing over a moving platform," Journal of Intelligent 8 Robotic Systems, vol. 79, no. 2, pp. 275-293, 2015.

[97] S. Zhang, W. Xiang, L. Zili, and M. Srinivasan, "Visual tracking of moving targets by freely flying honeybees," Visual neuroscience, vol. 4, no. 04, pp. 379-386, 1990.

[98] B. Hérissé, T. Hamel, R. Mahony, and F.-X. Russotto, "A terrainfollowing control approach for a vtol unmanned aerial vehicle using average optical flow," Autonomous robots, vol. 29, no. 3-4, pp. 381-399, 2010 .

[99] G. Portelli, J. Serres, F. Ruffier, and N. Franceschini, "Modelling honeybee visual guidance in a 3-d environment," Journal of Physiology-Paris, vol. 104, no. 1, pp. 27-39, 2010. 
[100] F. Expert and F. Ruffier, "Flying over uneven moving terrain based on optic-flow cues without any need for reference frames or accelerometers," Bioinspiration ES biomimetics, vol. 10, no. 2, p. 026003, 2015.

[101] C. Sabo, A. Cope, K. Gurny, E. Vasilaki, and J. A. Marshall, "Bioinspired visual navigation for a quadcopter using optic flow," in AIAA Infotech@Aerospace, 2016,p. 0404.

[102] D. Izzo and G. D. Croon, "Landing with time-to-contact and ventral optic flow estimates," Journal of Guidance, Control, and Dynamics, vol. 35, no. 4, pp. 1362-1367, 2012.

[103] F. Kendoul, "Four-dimensional guidance and control of movement using time-to-contact: Application to automated docking and landing of unmanned rotorcraft systems," The International Journal of Robotics Research, vol. 33, no. 2, pp. 237-267, 2014.

[104] M. T. Alkowatly, V. M. Becerra, and W. Holderbaum, "Body-centric modelling, identification, and acceleration tracking control of a quadrotor uav," International journal of modelling, identification and control, vol. 24, no. 1, pp. 29-41, 2015.

[105] G. C. de Croon, "Monocular distance estimation with optical flow maneuvers and efference copies: a stability-based strategy," Bioinspiration E biomimetics, vol. 11, no. 1, p. 016004, 2016.

[106] E. Baird, N. Boeddeker, M. R. Ibbotson, and M. V. Srinivasan, "A universal strategy for visually guided landing," Proceedings of the National Academy of Sciences, vol. 110, no. 46, pp. 18686-18691, 2013.

[107] P. Serra, R. Cunha, T. Hamel, D. Cabecinhas, and C. Silvestre, "Landing on a moving target using image-based visual servo control," in Decision and Control (CDC), 2014 IEEE 53rd Annual Conference on. IEEE, 2014, pp. 2179-2184.

[108] M. V. Srinivasan, "Going with the flow: a brief history of the study of the honeybee's navigational 'odometer'," Journal of Comparative Physiology $A$, vol. 200, no. 6 , pp. 563-573, 2014.

[109] N. Nourani-Vatani, J. Roberts, and M. V. Srinivasan, "Practical visual odometry for car-like vehicles," in Robotics and Automation, 2009. ICRA'09. IEEE International Conference on. IEEE, 2009, pp. 35513557. 
[110] H. Dahmen and H. A. Mallot, "Odometry for ground moving agents by optic flow recorded with optical mouse chips," Sensors, vol. 14, no. 11, pp. $21045-21064,2014$.

[111] R. Strydom, S. Thurrowgood, and M. Srinivasan, "Visual odometry: autonomous uav navigation using optic flow and stereo," in Australasian Conference on Robotics and Automation (ACRA), 2014, pp. 1-10.

[112] M. B. Milde, O. J. Bertrand, R. Benosmanz, M. Egelhaaf, and E. Chicca, "Bioinspired event-driven collision avoidance algorithm based on optic flow," in Event-based Control, Communication, and Signal Processing (EBCCSP), 2015 International Conference on. IEEE, 2015, pp. 1-7.

[113] A. Denuelle and M. V. Srinivasan, "Snapshot-based navigation for the guidance of uas," in Australasian Conference on Robotics and Automation (ACRA 2015), 2015.

[114] A. Briod, J.-C. Zufferey, and D. Floreano, "A method for ego-motion estimation in micro-hovering platforms flying in very cluttered environments," Autonomous Robots, vol. 40.

[115] S. Mafrica, S. Godiot, M. Menouni, M. Boyron, F. Expert, R. Juston, N. Marchand, F. Ruffier, and S. Viollet, "A bio-inspired analog silicon retina with michaelis-menten auto-adaptive pixels sensitive to small and large changes in light," Optics express, vol. 23, no. 5, pp. 5614-5635, 2015. 